\title{
Opportunities to use satellite technologies for asset condition monitoring of power networks under the European Space Agency's Integrated Applications Promotion programme
}

\author{
lan Downey ${ }^{1}$, Maria Segovia ${ }^{2}$, Chris D. Harrison ${ }^{3}$ \\ ${ }^{1}$ UK IAP Ambassador Platform, ESA, Harwell Campus, Didcot, Oxfordshire, UK \\ ${ }^{2} P N D C$, EEE Department, University of Strathclyde, Cumbernauld, Glasgow, UK \\ ${ }^{3}$ Energy Systems Catapult, Birmingham, UK \\ $凶$ E-mail: lan.downey@esa.int
}

\begin{abstract}
Network operators in the UK have stated their intention to target network reliability through improved procedures for asset management. This involves having a better understanding of how an asset deteriorates over time and their current health. The availability of good quality data is essential to this process. At the distribution level the majority of asset condition data comes from inspection, sometimes coupled with a form of continuous condition monitoring. Both are relatively expensive, with inspection also being very personnel-intensive. Satellites provide a variety of enabling capabilities that are applicable across many sectors. They can potentially support the inspection process of many of the assets present within distribution and transmission networks. This study presents an overview of potential satellite applications within the power industry, with particular emphasis on how they can be used for asset inspection and monitoring. A workshop was organised by European Space Agency and the Power Networks Demonstration Centre with UK network operators as participants to further explore some of the potential applications. The main areas identified are briefly outlined in this study.
\end{abstract}

\section{Introduction}

Electricity networks are identified as among the most vital of critical infrastructure sectors. Electrical power distribution is a critical input to almost all forms of economic and leisure activity. Disruption to supplies can have cascading effects throughout an entire economy and result in a significant loss of economic value by firms, governments, and households.

Network operators in the UK have stated their intention to target network reliability through improved procedures for asset management. This involves having a better understanding of how an asset deteriorates over time and their current health [1]. This will enable life in service to be predicted, thus the maintenance strategy can be optimised. The availability of good quality data is essential to this process.

Currently at the distribution level the majority of asset condition data comes from inspection, sometimes coupled with a form of continuous condition monitoring. Both are relatively expensive, with inspection also being very personnel-intensive. New technologies for data collection may reduce the number of person-hours associated with inspection and have the added benefit of allowing automated data processing and decision support. The European Space Agency (ESA) through the Advanced Research in Telecommunications Systems (ARTES) Integrated Applications Promotion (IAP) programme has supported several projects in this domain and is open to potential new activities [2].

Satellites provide a variety of enabling capabilities that are applicable across many sectors. They can potentially support the inspection process of many of the assets present within distribution and transmission networks. Previous analysis of this sector by ESA IAP suggested that costs due to network disruption are significant across the whole of Europe, split relatively evenly between firms and governments and households. Three generic potential areas of satellite applications were identified that could improve the reliability, robustness and resilience of electricity networks:

- The provision of satellite-based SCADA and GNSS applications combined with in-situ sensors to monitor network integrity in remote locations and allow early identification of possible component failures or structural fatigue.

- Satellite communications (SatCom) applications to provide network backup in the event of information technology (IT) and communications failures, and ensure a secure and reliable independent communications network controlled by electricity suppliers to promote network resilience.

- An integrated SatCom global navigation satellite system (GNSS) and earth observation (EO) solution to monitor extreme weather, ground motion and other geophysical events that could cause damage to or destruction of network infrastructure.

Consequently, ESA IAP held an open competition in 2013 on the topic of 'Maintenance and Recovery of High-Voltage Electricity Transport Systems' [3].

This paper presents an overview of potential satellite applications within the power industry, with particular emphasis on how they can be used for asset inspection and monitoring. These areas were identified through a joint workshop organised by the Power Networks Demonstration Centre (PNDC) and ESA to further explore and realise some of these potential applications. The PNDC, which has three UK network operators participating as members in its ongoing R\&D Programme, aims at accelerating the adoption of novel research and technologies in the electricity industry. 
The paper is organised as follows: First a description of the identified areas for satellite applications through the workshop is given, followed by a description of the available satellite technologies. The subsequent section provides an overview of the ESA IAP programme as well as some examples of the application projects that have been funded partly as a result of the 2013 competition. Finally, some conclusions and suggestions for future work are presented.

\section{Electricity networks challenges}

In May 2016 a workshop was jointly organised by the ESA and the PNDC, a research centre on Smart Grid applications which is part of the University of Strathclyde. Participants included representatives from the following UK transmission network operators (TNOs) and distribution network operators (DNOs): Scottish and Southern Electricity Networks, UK Power Networks, Scottish Power Energy Networks and the National Grid. In addition, other industrial and academic participants with expertise in satellite applications were invited. Five broad areas for possible further investigation were identified and are described below.

\subsection{Satellite observation for asset monitoring}

As mentioned in Section 1 the availability of good quality data is essential to the asset management process [1]. However, at the distribution level the majority of the condition data are collected through inspection, which is personnel-intensive and therefore expensive. Available satellite technology could provide information about critical assets reducing the number of inspections. A number of areas were identified where satellite observations could be used to aid condition monitoring, including:

- Monitoring the structural integrity of towers and poles.

- Monitoring subsidence around oil filled cables, particularly around the junction end boxes.

- Detection of cable theft.

- Power flow optimisation, potentially by dynamically rating overhead lines.

- Partial discharge detection.

- Lightning detection.

- Assessing areas prior to construction of critical assets, e.g. ground stability. Monitoring of structural integrity of assets during construction.

In addition, in a number of cases the possibility of using strategically placed sensors was suggested. This would allow both data sets to be combined to ascertain more information than would be available from the satellite observations alone, but with a lower number of sensors than would be required otherwise.

Although satellite observations alone or in combination with sensors have the potential to be applied to the above mentioned areas, a cost-benefit analysis needs to be done for each particular case. The availability of low cost sensors and the reduction of satellite observation cost should make their application cost effective.

\subsection{Satellite support for autonomous drones}

Currently unmanned aerial vehicles (UAVs) are being used, among other applications, for inspections of oil and gas infrastructure, wind turbines, and are starting to be applied for overhead line inspections. Satellites could support the use of UAVs for transmission and distribution network assets inspections in different ways. Particularly:

- Beyond visual line of sight (BVLOS) operation, enabling remote inspections. As an example, at present overhead line inspections carried out by UAVs need to be done within visual line of sight (VLOS) in the UK due to Civil Aviation Authority (CAA) regulations $[4,5]$. However, it is thought that the regulation will be relaxed in the near future for specific cases. The costs of VLOS UAV operations are broadly similar to the current practice, i.e. foot patrol plus helicopter. BVLOS UAV operations would cost less than current helicopter patrols due to lower mobilisation costs and the capacity to capture more data for analytics purposes.

- Combined Satellite and Drone Observations. Satellites could provide an initial assessment, e.g. in an emergency situation, when a fault has occurred. Drones could be sent afterwards for a detailed inspection on identified areas. Ensuring data/observations consistency is also a key consideration.

- Data transmission and feed into the asset management process. Data captured by drones and satellites could be transmitted via satellite and analysed, e.g. in the Cloud, before maintenance decisions are taken. This could be beneficial in remote or other areas of poor connectivity and provide more timely and resilient data capture and information dissemination depending on the costbenefit analysis.

\subsection{Storm prediction and monitoring for targeted recovery}

Accurate weather prediction is also an area of interest for the network operators. The information could be used to better predict renewables' production, for example. Particular focus was given to the forecasting of extreme weather events. Coupled with the identification of vulnerable areas (e.g. due to flooding) in the pre-event phase, evaluation of these particular areas before and after an extreme weather event could be carried out via satellites to assess damage. Weather data from satellites is already available at high temporal frequencies, currently up to $15 \mathrm{~min}$ intervals from geostationary platforms [6]. Updated forecasts would need to be available at similar temporal frequencies. This would assist, particularly:

- The ability to dynamically redeploy engineering resources in the field to areas of highest priority.

- Enabling better planning and adjustment of the renewables component in power grid balancing in light of weather extremes (solar, wind, wave etc.).

\subsection{Crew assistance to facilitate a more effective response}

Satellites could support crews in the field, particularly by:

- Improving communication with in-field workers as well as improving their security.

- Providing services to locate crews and assets deployed during ordinary maintenance and emergency activities.

This could help to save cost and increase team effectiveness. It is expected that satellite enabled communications can be used to provide enhanced and/or more resilient communication between local teams and the coordinator, particularly during emergency activities.

\subsection{Satellite communications for remote areas}

The number of communication data end points within the UK distribution grid is predicted to increase from 52,000 in 2011 to 695,000 in 2031 [7]. This increase is due to smarter monitoring and control requirements, including for asset condition monitoring. While generally these communications may be provided by land-based systems, there will be remote locations where land-based communications will not prove cost effective.

Satellite-based communications are currently used by all three DNOs represented at the workshop, however the main challenge in expanding their use is the cost. The cost associated with using the broadband type services currently deployed would be prohibitive 
for such a large number of endpoints. A secondary issue is that some locations, particularly valley locations in Scotland, do not have a clear view to the south.

In addition to data uses, the following possible voice communications applications were identified: black start assistance, mobile worker support (particularly for emergency communications) and construction phase communications.

\section{Use of satellites to tackle these challenges}

For the data communication applications, the evolution of new services to support infrastructure needs such as machine to machine, internet of things and smart and micro-grids are of growing interest to the space industry. Increasingly, it is anticipated that a hybrid telecommunications network will evolve based on merging terrestrial and satellite capabilities to ensure secure connectivity and accessibility. Low orbit mega constellations are already in prospect, which may provide a cost effective communication solution for many more applications in the medium to long term [8].

Satellite Navigation and positioning (SatNav, also generically known as GNSS) has become an essential service that is often taken for granted. It can provide precise time and position information anywhere on the globe with an accuracy ranging from millimetres to meters, depending on the SatNav technologies implemented. Besides the well-known Global Positioning System (GPS) system, a growing range of SatNav capability is being implemented, most notably via the deployment of Europe's Galileo system $[9,10]$. The Galileo programme is designed to be compatible with all existing and planned systems and interoperable with the American GPS and Russian Global Navigation Satellite System (referred to as GLONASS). In this sense, Galileo is positioned to enhance the coverage currently available, providing a more seamless and accurate experience for multi-constellation users around the world and supporting a wide range of existing and innovative new applications.

Satellite EO can acquire imagery globally, depending on the orbit of the respective satellite [11]. The coverage of EO is spatially continuous, and can help characterise the air and ground environment, including infrastructure built on the Earth's surface. EO has produced standardised and consistent data which have comparable over time and allowed change detection. The timely availability of space-borne information is conditioned by a number of factors, including the revisit time of the considered satellite(s) over a given point, the visibility (e.g. cloud cover for optical and IR observations) and the processing time.

Satellite data can be combined or complemented with in-situ data and airborne data to obtain the optimal combination of temporal and spatial resolution, and eventually provide the information needed in a timely manner. Among airborne platforms, UAVs might be an alternative to manned aircraft systems. Operating UAVs may also represent a cost advantage relative to conventional aircraft.

In addition, space robotic technologies and data processing technologies (e.g. data compression, anomaly detection algorithms etc.) developed in the context of satellite operations may be of interest and utility.

\section{ESA IAP programme and examples}

The IAP focuses on the development and pilot operations of a range of new and innovative solutions by using and integrating different available space assets with terrestrial assets, resulting in viable and sustainable services for the benefit of society [2]. The IAP programme is dedicated to the development, implementation and pilot operations of integrated applications based on the demand of users and targeting sustainable operational services. The goal is to provide innovative, added value services by combining different space assets, such as telecommunications, earth observation, navigation, and human spaceflight technologies, integrating them with existing terrestrial assets and legacy systems.
The ARTES IAP projects cover feasibility studies and demonstration projects [2]. Any organisation can propose to develop a new commercially promising space-based application or service through a process of direct negotiation. The programme offers matched funding of up to $75 \%$ (in case of small and medium enterprises and depending on Member State delegations approval). Occasionally $100 \%$ funded feasibility study competitive opportunities are offered.

Energy security is critical for the growth and prosperity of European economies. Not supplying electricity to consumers results in revenue losses for electricity producers, transmission operators and distribution operators. Every year, tens of millions of Euros of 'non-distributed electricity' are lost by transmission system operators (TSOs) and distribution system operators (DSOs) essentially as a result of structural defects on the transmission network. This number can increase to hundreds of millions of Euros as a result of natural disasters causing heavy damages to the infrastructure. In addition to losing revenue, TSOs \& DSOs have to pay penalties to the electricity producers and to their major customers. Those penalties can amount to several hundreds of thousands of Euros per day and per customer. Space-based systems and services already play an important part in the commercial sectors of the energy industry, but there is still a significant potential for increasing this.

Space services have applications in renewable energy systems as well as in traditional oil-carbon and nuclear sectors. Such services can deliver benefits throughout all the phases of energy production and supply, ranging from identifying reservoirs and sites, to controlling and monitoring the performance and integrity of distribution networks across Europe. They also provide support to policy formulation and enforcement.

A variety of applications projects in the power sector have been developed under IAP, some examples of which are given below.

\subsection{SharperSat}

The purpose of the SharperSat project, implemented in Finland, was to design, develop and validate an integrated solution for TSOs and DSOs [12]. The aim was to significantly improve the maintenance and recovery of their overhead assets and reduces related operational costs. The needs defined by end users are

- Rapid recognition of damages after a storm.

- Clearance need detection and prioritisation of areas.

- Supervision of vegetation clearance.

- Cost efficiency of new methods.

To meet user needs Sharper Shape has used satellite EO supported by remote sensing and airborne data gathered by UAVs and its own software solutions to provide a new level of accuracy and faster results. Software automatically analyses data and identifies the need for corrective actions in the field. The service integrates with the end users producing a maintenance plan which is economic optimised.

The rationale behind the service concept is to provide reductions in operational expenditure. This is achieved through always using the most economic method for the initial analysis which is then used to determine where more expensive and accurate inspection methods should be deployed to minimise unnecessary inspection and maintenance work.

\subsection{Gridwatch}

GridWatch is a project based in Italy aimed at potential customers in both transmission and distribution, who are in charge of delivering electrical energy along overhead lines [13]. Identified user needs concern issues around the integrity and operating conditions they face during normal operations. Particular user needs include:

- Reception of information on pylon movements due to terrain subsidence. 
- Knowledge of the condition of the pylons in extreme events (earthquakes, floods etc.) within $24 \mathrm{~h}$.

- Assessment of the dynamic capacity of their lines to avoid overloading.

The proposed service is composed of two elements, each addressing specific departments of the TSOs:

- Maintenance of the power infrastructure.

- Network operations and energy dispatching.

The maintenance service provides the maintenance department with information about key parameters of the monitored overhead lines, without the need of site inspections. Information is provided several times per day, and allows the user to enhance the current inspection procedures, characterised by two to three site inspections per year.

The network service provides information about line transmission capacities, to support the energy dispatch operations in critical conditions such as line overloading. Such criticalities may occur in different situations such as power lines outages or in proximity of renewable energy sources due to unpredictable production peaks, which are potential risks for the TSOs that need to guarantee power supplies within safe operational limitations. The proposed solution overcomes the current conservative approach based on static estimations of network capacity by providing local and adjusted parameters that can be used to evaluate actual network capacity and so determine the power that can be safely transmitted at a particular time.

\subsection{HV SatProtec}

This project is a Swiss-German collaboration also focused on service delivery to TSOs and DSOs [14].

Two HV-SATPROTEC services have been developed, which are based on the integration of different space and non-space assets to provide users with systematic and timely information on the state of the infrastructures and on potential threats in their surroundings at lower costs. These services are aimed at supporting a more cost-efficient planning of necessary maintenance tasks, anticipate risks threatening overhead power lines and improve the overall safety of monitoring operations within transmission corridors. This will help to reduce the risk of power outages and to decrease the related high maintenance/recovery operation costs.

\section{Conclusions and future work}

Network operators in the UK have stated their intention to target network reliability through improved procedures and better understanding of asset condition management.

Satellites provide a variety of enabling capabilities that are applicable to the power and energy domain and many other sectors. They can potentially support the asset inspection process within both the distribution and transmission networks.

A workshop, which included participants from UK network operators, was organised by ESA and the PNDC to explore potential applications. The main areas identified for further investigation were:

- Satellite Observation for Asset Monitoring

- Satellite Support for Autonomous Drones.

- Storm Prediction and Monitoring for Targeted Recovery.

- Crew Assistance to Facilitate a More Effective Response.

- Satellite Communications for Remote Areas.

An overview of potential satellite solutions that could help address these areas of interest has been presented above.

This paper has also described the ARTES IAP programme funded by ESA. The IAP focuses on the development and pilot operations of a range of new and innovative solutions by using and integrating different available space assets with terrestrial assets, resulting in viable and sustainable services for the benefit of society.

A variety of ESA IAP projects have been developed to address industry challenges in the Energy sector. Some of these projects have been outlined in this paper.

As future work, some of the ideas from the workshop are being developed by PNDC, with funding from the ARTES IAP programme and other sources likely to be sorted. This is being done in collaboration with UK network operators and other interested parties. Further partners are welcomed both for existing and potential new streams of work.

The IAP programme is continuously open to additional potential new activities from UK entities in the power and energy industries.

\section{References}

1 Vachtsevanos, G., Lewis, F.L., Roemer, M., et al: 'Intelligent fault diagnosis and prognosis for engineering systems' (Wiley, 2006)

2 The Artes application. Available at https://artes-apps.esa.int/

3 Maintenance and recovery of high voltage electricity transport systems, 2013. Available at https://artes-apps.esa.int/opportunities/invitation-to-tender/ maintenance-and-recovery-high-voltage-electricity-transport-systems

4 The Air Navigation Order 2016, Stautory Instruments 765, UK Government, 2016

5 Unmanned Aircraft, Requirements for operating in airspace (Civil Aviation Authority, UK, 2015). Available at www.caa.co.uk/Commercial-industry/ Aircraft/Unmanned-aircraft/Unmanned-Aircraft/

6 Monitoring weather and climate from space. Available at http://www.eumetsat.int website/home/Satellites/CurrentSatellites/Meteosat/index.html

7 Hulme, A., Sennes, V.: 'DNO - Smart Grid Communications Requirements' (Energy Networks Association, London, 2011). Available at www. energynetworks.org/assets/files/electricity/engineering/telecoms/eitc/restricted/ Reference\%20Doc/Telent.pdf

8 Alvarez, J., Walls, B.: 'Constellations, clusters, and communication technology: expanding small satellite access to space'. 2016 IEEE Aerospace Conf. Big Sky, MT, 2016, pp. 1

9 'What is Galileo?' (European Space Agency, 2016). Available at www.esa.int Our_Activities/Navigation/Galileo/What_is_Galileo

10 Nurmi, J, Lohan, E.S., Sand, S., et al.: 'GALILEO positioning technology' (Springer, London, UK, 2015

11 Belward, A.S., Skøien, J.O.: 'Who launched what, when and why; trends in global land-cover observation capacity from civilian earth observation satellite', ISPRS J. Photogramm. Remote Sens., 2015, 103, pp. 115-128

12 Sharpersat - Maintenance and recovery of high voltage electricity transport system. Available at https://artes-apps.esa.int/projects/sharpersat

13 Gridwatch - Maintenance and recovery of high voltage electricity transport system. Available at https://artes-apps.esa.int/projects/gridwatch

14 HV-Satprotect - Maintenance and recovery of high voltage electricity transport system. Available at https://artes-apps.esa.int/projects/hv-satprotec 